\title{
ASPECTS OF NEUTRINO MASS MATRICES
}

\author{
P.H. FRAMPTON \\ Department of Physics and Astronomy, \\ University of North Carolina at Chapel Hill, \\ Chapel Hill, NC 27599-3255. \\ E-mail:frampton@physics.unc.edu
}

\begin{abstract}
After an Introduction briefly describing the rise and fall of the three-zero texture of the Zee model, we discuss still-allowed two-zero textures for the Majorana threeneutrino mass matrix. Finally, a model with two right-handed neutrinos and two Dirac texture zeros is described (FGY model) which can relate CP violation in leptogenesis to $\mathrm{CP}$ violation in long-baseline neutrino oscillations.
\end{abstract}

\section{Introduction}

The minimal standard model involves three chiral neutrino states, but it does not admit renormalizable interactions that can generate neutrino masses. Nevertheless, experimental evidence show that both solar and atmospheric neutrinos display flavor oscillations, and hence that neutrinos do have mass.

Among the many renormalizable and gauge-invariant extensions of the standard model that can provide neutrino masses are:

- The introduction of a complex triplet of mesons $\left(T^{++}, T^{+}, T^{0}\right)$ coupled bilinearly to pairs of lepton doublets ${ }^{1}$. They must also couple bilinearly to the Higgs doublet(s) so as to avoid spontaneous $B-L$ violation and the appearance of a massless and experimentally excluded majoron. This mechanism can generate an arbitrary complex symmetric Majorana mass matrix for neutrinos.

- The introduction of singlet counterparts to the neutrinos with very large Majorana masses. The interplay between these mass terms and those generated by the Higgs boson - the so-called see-saw mechanism - yields an arbitrary but naturally small Majorana neutrino mass matrix.

- The introduction of a charged singlet meson $f^{+}$coupled antisym- 
metrically to pairs of lepton doublets, and a doubly-charged singlet meson $g^{++}$coupled bilinearly both to pairs of lepton singlets and to pairs of $f$-mesons. An arbitrary Majorana neutrino mass matrix is generated in two loops.

- The introduction of a charged singlet meson $f^{+}$coupled antisymmetrically to pairs of lepton doublets and (also antisymmetrically) to a pair of Higgs doublets. This simple mechanism was first proposed by Zee ${ }^{2}$, and results (at one loop) in a Majorana mass matrix in the flavor basis $(e, \mu, \tau)$ of a special form:

$$
\mathbf{M}=\left(\begin{array}{ccc}
0 & m_{e \mu} & m_{e \tau} \\
m_{e \mu} & 0 & m_{\mu \tau} \\
m_{e \tau} & m_{\mu \tau} & 0
\end{array}\right)
$$

Because the diagonal entries of $\mathbf{M}$ are zero, the amplitude for noneutrino double beta decay vanishes at lowest order and this process cannot proceed at an observable rate. Furthermore, the parameters $m_{e \mu}, m_{e \tau}$ and $m_{\mu \tau}$ may be taken real and non-negative without loss of generality, whence $\mathbf{M}$ becomes real as well as traceless and symmetric. With this convention, the analog to the Kobayashi-Maskawa matrix becomes orthogonal: $\mathbf{M}$ is explicitly $\mathrm{CP}$ invariant and $\delta=0$.

The sum of the neutrino masses (the eigenvalues of $\mathbf{M}$ ) vanishes: $m_{1}+$ $m_{2}+m_{3}=0$. When the squared-mass hierarchy is taken into account, that the solar squared mass difference is very much smaller than the atmospheric squared mass difference, there are two possibilities. In case A, we have $m_{1}+$ $m_{2}=0$ and $m_{3}=0$. This case arises iff at least one of the three parameters in $\mathbf{M}$ vanishes. In case $\mathbf{B}$, we have $m_{1}=m_{2}$ and $m_{3}=-2 m_{2}>0$. It has been shown ${ }^{3,4}$ that neither case is consistent with the SuperKamiokande and SNO data so the the Zee model is ruled out by these experiments.

\section{Two-Zero Textures}

Having excluded one three-zero texture we now study two-zero possibilities.

Quark masses and mixings are described by two $3 \times 3$ matrices which involve a total of ten convention-independent real quantities: four KobayashiMaskawa parameters and six positive quark masses. The lepton sector requires only three additional parameters (the charged lepton masses) in the 
minimal - and manifestly incomplete - standard model wherein neutrinos are massless.

We assume that neutrino phenomenology can be formulated in terms of three left-handed neutrino states with a complex symmetric Majorana mass matrix $\mathbf{M}$. We do not commit ourselves to any particular mechanism by which neutrino masses are generated. Of the twelve real parameters characterizing $\mathbf{M}$, three are arbitrary phases of the three flavor eigenstates. Thus the neutrino sector involves nine convention-independent parameters. The two squared-mass differences and the four Kobayashi-Maskawa analogs are likely to be measureable in practice. So also is the quantity $\left|\mathbf{M}_{e e}\right|$ through the search for neutrinoless double beta decay. There remain two parameters that are measureable in principle but apparently not easily in practice.

It will be useful to define the ratio of squared-mass differences, whose estimated value is: $R_{\nu} \equiv \frac{\Delta_{s}}{\Delta_{a}} \approx 2 \times 10^{-2}$.

We turn to the question of which two independent entries of $\mathbf{M}$ can vanish in the basis wherein the charged lepton mass matrix is diagonal. Of the fifteen logical possibilities, we find just seven to be in accord with our empirical hypotheses. We discuss them individually, with the nonvanishing entries in each case denoted by $X$ 's. Our results are presented to leading order in the small parameter $s_{2}$. We begin with a texture in which $\mathbf{M}_{e e}=\mathbf{M}_{e \mu}=0$ :

Case $A_{1}: \quad\left(\begin{array}{ccc}0 & 0 & X \\ 0 & X & X \\ X & X & X\end{array}\right)$

For this texture, $\mathbf{M}_{e e}=0$ so that the amplitude for no-neutrino double beta decay vanishes to lowest order in neutrino masses. If Case $A_{1}$ is realized in nature, the neutrinoless process simply cannot be detected. There is even more to say. Two of the squared neutrino masses are suppressed relative to the third by a factor of $s_{2}^{2}$. As a result we find that $s_{2}$ can lie close to its present experimental upper limit. This prediction will become more precise when $\theta_{3}$ is better measured. However, the CP-violating parameter $\delta$ is entirely unconstrained. For case $A_{1}$ the subdominant angle $\theta_{2}$ is likely to be measureable and neutrinos may display observable $\mathrm{CP}$ violation.

Case $A_{2}: \quad\left(\begin{array}{ccc}0 & X & 0 \\ X & X & X \\ 0 & X & X\end{array}\right)$

This texture has $\mathbf{M}_{e e}=\mathbf{M}_{e \tau}=0$. Its phenomenological consequences are 
nearly the same as those of Case $A_{1}$.

Case $B_{1}: \quad\left(\begin{array}{ccc}X & X & 0 \\ X & 0 & X \\ 0 & X & X\end{array}\right)$

With $\mathbf{M}_{\mu \mu}=\mathbf{M}_{e \tau}=0$, we find an acceptable solution if and only if $\left|s_{2} \cos \delta \tan 2 \theta_{1}\right| \ll 1$, in which case the three neutrinos are nearly degenerate in magnitude because $t_{1}^{2} \simeq 1$. The texture is also promising in regard to the search for neutrinoless double beta decay. We obtain $\mathbf{M}_{e e} \simeq-t_{1}^{2} \sqrt{\Delta_{a} /\left|1-t_{1}^{4}\right|}$ likely to exceed $100 \mathrm{meV}$. The rate of neutrinoless double beta decay may approach its current experimental upper limit.

Case $B_{2}: \quad\left(\begin{array}{ccc}X & 0 & X \\ 0 & X & X \\ X & X & 0\end{array}\right)$

This texture, with $\mathbf{M}_{\tau \tau}=\mathbf{M}_{e \mu}=0$, is as in $B_{1}$ with $t_{1}$ replaced by $-1 / t_{1}$. Its phenomenological consequences are nearly the same as those of Case $B_{1}$.

Case $B_{3}:\left(\begin{array}{ccc}X & 0 & X \\ 0 & 0 & X \\ X & X & X\end{array}\right)$ and Case $B_{4}: \quad\left(\begin{array}{ccc}X & X & 0 \\ X & X & X \\ 0 & X & 0\end{array}\right)$

The phenomenological implications of all four cases $B_{i}(\mathrm{i}=1,2,3,4)$ are substantially the same.

Case $C: \quad\left(\begin{array}{ccc}X & X & X \\ X & 0 & X \\ X & X & 0\end{array}\right)$

Our seventh and last allowed texture has $\mathbf{M}_{\mu \mu}=\mathbf{M}_{\tau \tau}=0$.

No other two-zero texture of the neutrino mass matrix is compatible with our empirical hypotheses. It is easily verified that no two of our allowed two-zero textures can be simultaneously satisfied while remaining consistent with our empirical hypotheses. It follows that there is no tolerable threezero texture.

The seven allowed two-zero neutrino textures fall into three classes: $A$ (with two members), $B$ (with four members), and $C$. The textures within each class are difficult or impossible to distinguish experimentally, but each of the three classes has radically different implications. For class $A$, the 
subdominant angle $\theta_{2}$ is expected to be relatively large, but no-neutrino $\beta \beta$ decay is forbidden. For class $B$, the latter process should be measureable by the next generation of double beta decay experiments, whilst $s_{2}$ may or may not be large enough to be detected. However, if $s_{2}$ is comparable to its experimental upper limit, $\mathrm{CP}$ violation must be nearly maximal and should be readily detectable by proposed experiments. For class $C$, no-neutrino $\beta \beta$ decay is likely to be observable and $\theta_{2}$ ought to be large enough to measure and to permit a search for $\mathrm{CP}$ violation. It is surprising that such a great variety of textures of the neutrino mass matrix can fit what is presently known about neutrino masses and oscillations. Future data should reveal which, if any, of these textures should serve as a guide to the model builder.

\section{FGY Model}

One of the most profound ideas is ${ }^{6}$ that baryon number asymmetry arises in the early universe because of processes which violate $\mathrm{CP}$ symmetry and that terrestrial experiments on $\mathrm{CP}$ violation could therefore inform us of the details of such cosmological baryogenesis.

The early discussions of baryogenesis focused on the violation of baryon number and its possible relation to proton decay. In the light of present evidence for neutrino masses and oscillations it is more fruitful to associate the baryon number of the universe with violation of lepton number ${ }^{7}$. In the present Letter we shall show how, in one class of models, the sign of the baryon number of the universe correlates with the results of $\mathrm{CP}$ violation in neutrino oscillation experiments which will be performed in the forseeable future.

Present data on atmospheric and solar neutrinos suggest that there are respective squared mass differences $\Delta_{a} \simeq 3 \times 10^{-3} \mathrm{eV}^{2}$ and $\Delta_{s} \simeq 5 \times$ $10^{-5} \mathrm{eV}^{2}$. The corresponding mixing angles $\theta_{1}$ and $\theta_{3}$ satisfy $\tan ^{2} \theta_{1} \simeq 1$ and $0.6 \leq \sin ^{2} 2 \theta_{3} \leq 0.96$ with $\sin ^{2} \theta_{3}=0.8$ as the best fit. The third mixing angle is much smaller than the other two, since the data require $\sin ^{2} 2 \theta_{2} \leq 0.1$.

A first requirement is that our model ${ }^{5}$ accommodate these experimental facts at low energy.

In the minimal standard model, neutrinos are massless. The most economical addition to the standard model which accommodates both neutrino masses and allows the violation of lepton number to underly the cosmological baryon asymmetry is two right-handed neutrinos $N_{1,2}$.

These lead to new terms in the lagrangian: 


$$
\begin{aligned}
\mathbf{L} & =\frac{1}{2}\left(N_{1}, N_{2}\right)\left(\begin{array}{cc}
M_{1} & 0 \\
0 & M_{2}
\end{array}\right)\left(\begin{array}{l}
N_{1} \\
N_{2}
\end{array}\right)+ \\
& +\left(N_{1}, N_{2}\right)\left(\begin{array}{ccc}
a & a^{\prime} & 0 \\
0 & b & b^{\prime}
\end{array}\right)\left(\begin{array}{l}
l_{1} \\
l_{2} \\
l_{3}
\end{array}\right) H+\text { h.c. }
\end{aligned}
$$

where we shall denote the rectangular Dirac mass matrix by $D_{i j}$. We have assumed a texture for $D_{i j}$ in which the upper right and lower left entries vanish. The remaining parameters in our model are both necessary and sufficient to account for the data.

For the light neutrinos, the see-saw mechanism leads to the mass matrix ${ }^{8}$

$$
\begin{aligned}
\hat{L} & =D^{T} M^{-1} D \\
& =\left(\begin{array}{ccc}
\frac{a^{2}}{M_{1}} & \frac{a a^{\prime}}{M_{1}} & 0 \\
\frac{a a^{\prime}}{M_{1}} & \frac{\left(a^{\prime}\right)^{2}}{M_{1}}+\frac{b^{2}}{M_{2}} & \frac{b b^{\prime}}{M_{2}} \\
0 & \frac{b b^{\prime}}{M_{2}} & \frac{\left(b^{\prime}\right)^{2}}{M_{2}}
\end{array}\right)
\end{aligned}
$$

We take a basis where $a, b, b^{\prime}$ are real and where $a^{\prime}$ is complex $a^{\prime} \equiv$ $\left|a^{\prime}\right| e^{i \delta}$. To check consistency with low-energy phenomenology we temporarily take the specific values (these will be loosened later) $b^{\prime}=b$ and $a^{\prime}=\sqrt{2} a$ and all parameters real. In that case:

$$
\hat{L}=\left(\begin{array}{ccc}
\frac{a^{2}}{M_{1}} & \frac{\sqrt{2} a^{2}}{M_{1}} & 0 \\
\frac{\sqrt{2} a^{2}}{M_{1}} & \frac{2 a^{2}}{M_{1}}+\frac{b^{2}}{M_{2}} & \frac{b^{2}}{M_{2}} \\
0 & \frac{b^{2}}{M_{2}} & \frac{b^{2}}{M_{2}}
\end{array}\right)
$$

We now diagonalize to the mass basis by writing:

$$
\mathbf{L}=\frac{1}{2} \nu^{T} \hat{L} \nu=\frac{1}{2} \nu^{\prime T} U^{T} \hat{L} U \nu^{\prime}
$$

where

$$
\begin{aligned}
U & =\left(\begin{array}{ccc}
1 / \sqrt{2} & 1 / \sqrt{2} & 0 \\
-1 / 2 & 1 / 2 & 1 / \sqrt{2} \\
1 / 2 & -1 / 2 & 1 / \sqrt{2}
\end{array}\right) \times \\
& \times\left(\begin{array}{ccc}
1 & 0 & 0 \\
0 & \cos \theta & \sin \theta \\
0 & -\sin \theta & \cos \theta
\end{array}\right)
\end{aligned}
$$

We deduce that the mass eigenvalues and $\theta$ are given by

$$
m\left(\nu_{3}^{\prime}\right) \simeq 2 b^{2} / M_{2} ; \quad m\left(\nu_{2}^{\prime}\right) \simeq 2 a^{2} / M_{1} ; \quad m\left(\nu_{1}^{\prime}\right)=0
$$


and

$$
\theta \simeq m\left(\nu_{2}^{\prime}\right) /\left(\sqrt{2} m\left(\nu_{3}^{\prime}\right)\right)
$$

in which it was assumed that $a^{2} / M_{1} \ll b^{2} / M_{2}$.

By examining the relation between the three mass eigenstates and the corresponding flavor eigenstates we find that for the unitary matrix relevant to neutrino oscillations that

$$
U_{e 3} \simeq \sin \theta / \sqrt{2} \simeq m\left(\nu_{2}\right) /\left(2 m\left(\nu_{3}\right)\right)
$$

Thus the assumptions $a^{\prime}=\sqrt{2} a, b^{\prime}=b$ adequately fit the experimental data, but $a^{\prime}$ and $b^{\prime}$ could be varied around $\sqrt{2} a$ and $b$ respectively to achieve better fits.

But we may conclude that

$$
\begin{aligned}
& 2 b^{2} / M_{2} \simeq 0.05 \mathrm{eV}=\sqrt{\Delta_{a}} \\
& 2 a^{2} / M_{1} \simeq 7 \times 10^{-3} \mathrm{eV}=\sqrt{\Delta_{s}}
\end{aligned}
$$

It follows from these values that $N_{1}$ decay satisfies the out-of-equilibrium condition for leptogenesis (the absolute requirement is $m<10^{-2} \mathrm{eV}^{9}$ ) while $N_{2}$ decay does not. This fact enables us to predict the sign of CP violation in neutrino oscillations without ambiguity.

Let us now come to the main result. Having a model consistent with all low-energy data and with adequate texture zeros ${ }^{10}$ in $\hat{L}$ and equivalently $D$ we can compute the sign both of the high-energy CP violating parameter $\left(\xi_{H}\right)$ appearing in leptogenesis and of the $\mathrm{CP}$ violation parameter which will be measured in low-energy $\nu$ oscillations $\left(\xi_{L}\right)$.

We find the baryon number $B$ of the universe produced by $N_{1}$ decay proportional to ${ }^{11}$

$$
\begin{aligned}
B & \propto \xi_{H}=\left(\operatorname{ImD} D^{\dagger}\right)_{12}^{2}=\operatorname{Im}\left(a^{\prime} b\right)^{2} \\
& =+Y^{2} a^{2} b^{2} \sin 2 \delta
\end{aligned}
$$

in which $B$ is positive by observation of the universe. Here we have loosened our assumption about $a^{\prime}$ to $a^{\prime}=Y a e^{i \delta}$.

At low energy the $\mathrm{CP}$ violation in neutrino oscillations is governed by the quantity

$$
\xi_{L}=\operatorname{Im}\left(h_{12} h_{23} h_{31}\right)
$$

where $h=\hat{L} \hat{L}^{\dagger}$. 
Using Eq.(3) we find:

$$
\begin{aligned}
& h_{12}=\left(\frac{a^{3} a^{\prime *}}{M_{1}^{2}}+\frac{a\left|a^{\prime}\right|^{2} a^{\prime *}}{M_{1}^{2}}\right)+\frac{a a^{\prime} b^{2}}{M_{1} M_{2}} \\
& h_{23}=\left(\frac{b b^{\prime} a^{\prime 2}}{M_{1} M_{2}}\right)+\left(\frac{b^{3} b^{\prime}}{M_{2}^{2}}+\frac{b b^{\prime 3}}{M_{2}^{2}}\right) \\
& h_{31}=\left(\frac{a a^{\prime *} b b^{\prime}}{M_{1} M_{2}}\right)
\end{aligned}
$$

from which it follows that

$$
\xi_{L}=-\frac{a^{6} b^{6}}{M_{1}^{3} M_{2}^{3}} \sin 2 \delta\left[Y^{2}\left(2+Y^{2}\right)\right]
$$

Here we have taken $b=b^{\prime}$ because the mixing for the atmospheric neutrinos is almost maximal.

Neutrinoless double beta decay $(\beta \beta)_{0 \nu}$ is predicted at a rate corresponding to $\hat{L}_{e e} \simeq 3 \times 10^{-3} \mathrm{eV}$.

The comparison between Eq.(11) and Eq.(14) now gives a unique relation between the signs of $\xi_{L}$ and $\xi_{H}$.

This fulfils in such a class of models the idea of ${ }^{6}$ with only the small change that baryon number violation is replaced by lepton number violation.

\section{Acknowledgement}

I wish to thank Ruth Daly, Tom Kephart, the late Behram Kursunoglu, Steve Mintz, Arnold Perlmutter and Ina Sarcevic for organizing the very memorable Fest. This work was supported in part by the Department of Energy under Grant Number DE-FG02-97ER-410236.

\section{References}

1. G. Lazarides, Q. Shafi and C. Wetterich, Nucl. Phys. B181, 287 (1981); R.N. Mohapatra and G. Senjanović, Phys. Rev. D23, 165 (1981); C. Wetterich, Nucl. Phys. B187, 343 (1981).

2. A. Zee, Phys. Lett, 93B 389 (1980).

3. P.H. Frampton and S.L. Glashow, Phys. Lett. B461, 95 (1999). hep-ph/9906375.

4. P.H. Frampton, M.C. Oh and T. Yoshikawa, Phys. Rev. B65, 073014 (2002). hep-ph/0110300. 
5. P.H. Frampton, S.L. Glashow and T. Yanagida, Phys. Lett. B548, 119 (2002). hep-ph/0208157

6. A.D. Sakharov, Pis'ma Zh. Eksp. Teor. Fiz. 5, 32 (1967) [JETP Lett. 5, 24 (1967)]

7. M. Fukugita and Y. Yanagida, Phys. Lett. B174, 45 (1986).

8. T. Yanagida, in Proceedings of the Workshop on Unified Theories and Baryon Number of the Universe Editors:O.Sawada and A. Sugamoto, (Tsukuba, Japan, 1979), KEK Report KEK-79-18, page 95.

S.L. Glashow, Cargese lectures (1979).

M. Gell-Mann, P. Ramond and R. Slansky, in Supergravity Editors: D.Z. Freedman and P. Van Nieuwenhuizen, (North-Holland, Amsterdam, 1979).

9. W. Buchmüller, in Proceedings of the Eighth Symposium on Particles, Strings and Cosmology. Editors: P.H. Frampton and Y.J. Ng. Rinton Press, New Jersey (2001), page 97.

10. P.H. Frampton, S.L. Glashow and D. Marfatia, Phys. Lett. B536, 79 (2002).

11. See e.g. W. Buchmüller and M. Plumacher, Phys. Reps. 320, 329 (1999), and references therein. 